\title{
Electron Energy Loss Spectroscopy of Single Silicon Nanocrystals: The Conduction Band
}

\author{
P. E. Batson and J. R. Heath \\ IBM T.J. Watson Research Center, Box 218, Yorktown Heights, New York 10598
}

(Received 20 May 1993)

\begin{abstract}
Spatially resolved electron energy loss spectroscopy has been performed on single, $\mathrm{H}$-terminated, $\mathrm{Si}$ nanocrystals in the size range 25-500 $\AA$. The particles were prepared via the gas-phase photolysis of a dilute $\mathrm{Si}_{2} \mathrm{H}_{6} / \mathrm{He}$ mixture in a gas flow cell, and deposited on a holey carbon grid for analysis. Energy loss within a few $\mathrm{eV}$ of the core $2 p$ ionization edge reveals information about the conduction band states at $\Delta_{1}$ and $L_{1}$ in the Brillouin zone. The conduction band edge is observed to shift to higher energy as the inverse square of the particle radius. In addition, a strong increase in the oscillator strength for these transitions is observed for decreasing particle sizes below $60 \AA$.
\end{abstract}

PACS numbers: 73.20.Dx, 78.55.Hx, 79.20.-m

Silicon nanostructures in the quantum confined size range have received quite a bit of recent attention. This is, in large part, due to the observation that they efficiently photoluminesce in the visible. Si is an indirect gap material, and thus, photoemission across the band gap is quantum mechanically forbidden. However, strong luminescence has been observed in systems such as $\mathrm{Si}$ nanocrystals in an oxide matrix [1], O-terminated $\mathrm{Si}$ nanocrystals [2], H-terminated silicon nanocrystals [3], and "porous silicon" [4], which is well described as a matrix of silicon quantum structures. The mechanism for this luminescence has been investigated theoretically by a number of groups [5-8], although a clear consensus for the appropriate model has not yet emerged. A major impediment toward developing such a model is the lack of experimental data which relate the size and the shape of a $\mathrm{Si}$ quantum structure to spectroscopic observables. In this Letter we attempt to fill this void by recording electron energy loss spectra (EELS) from single H-terminated Si nanocrystals. Spectroscopic features near the $2 p$ core ionization edge yield information concerning conduction band states near $\Delta_{1}$ and $L_{1}$ in the Brillouin zone. This energy-loss near-edge structure (ELNES) is measured as a function of particle size in the range from 500 to $25 \AA$. ELNES reveals that the conduction band structure of the particles remains nearly bulklike for particles as small as $80-90 \AA$. As the particle size decreases, the relative amounts of scattering associated with $\Delta_{1}$ and $L_{1}$ change, although in a manner which is consistent with that previously observed for thin, flat samples [9]. For sizes below $50 \AA$, the conduction band edge shape takes on a single band-edge character, shifts to higher energies as the inverse square of the particle radius, and displays a large increase in oscillator strength.

$\mathrm{H}$-terminated $\mathrm{Si}$ nanoparticles were prepared in a manner similar to that described by Jasinski and LeGoues [10], although the apparatus used here has been substantially modified from the one described in their work. The particles are synthesized via ArF excimer laser $(h v=6.4 \mathrm{eV})$ photolysis of a dilute $\mathrm{Si}_{2} \mathrm{H}_{6} / \mathrm{He} \mathrm{mix-}$ ture. The cell is approximately $0.75 \mathrm{~m}$ long and is equipped with a Brewster's angle UV quartz entrance window. He [100 SCCM (cubic centimeters per minute at standard temperature and pressure) flow] enters the cell near the quartz window. Approximately $5 \mathrm{~cm}$ downstream of the quartz window a $5 \% \mathrm{Si}_{2} \mathrm{H}_{6}$ in He mixture enters the cell (3-10 SCCM flow). The $\mathrm{He} / \mathrm{Si}_{2} \mathrm{H}_{6}$ mixture enters a "stirred" $25 \mathrm{~cm}$ long region of the cell. This region consists of a $2.5 \mathrm{~cm}$ diam stainless tube mounted at either end on rotary bearings. It is equipped with three stainless blades which are symmetrically mounted on the interior walls of the tube and run along the entire axis. A beveled gear is mounted about the outer diameter at the middle of the tube, and a magnetically coupled rotary feedthrough connects to the gear and spins the tube at $300-500 \mathrm{rpm}$. The rotating blades assure good mixing of the gas-phase reagents. Supports for microscope grids or some other substrate are placed downstream of the stirred region. Approximately $10 \mathrm{~cm}$ downstream of these supports the gas flow is directed through a pumpout tube equipped with a throttle valve. During particle synthesis the pressure of the tube is maintained at 5 Torr. The ArF laser is directed down the entire axis of the flow cell, and is operated at $50 \mathrm{~Hz}$ and $300 \mathrm{~mJ} /$ pulse.

Infrared absorption spectroscopy of particles deposited on an intrinsic $\mathrm{Si}$ wafer indicates that the particles are $\mathrm{H}$ terminated, with no features ascribable to $\mathrm{Si}-\mathrm{O}$ initially present. Absorption features near $1060 \mathrm{~cm}^{-1}$ do appear if the particles are exposed to air for a period of several minutes. EELS measurements showed surface plasmon resonances ranging from 9.2 to $10.6 \mathrm{eV}$ depending on the probe position. Expected values lie between $9.6 \mathrm{eV}$ $(l=1)$ and $11.8 \mathrm{eV}(l=\infty)$ for the clean silicon/vacuum interface. In addition, no oxide-related structure in the 105-115 eV energy loss range was observed, ruling out the presence of siloxene structure.

Of some concern here is the effect of a H-terminated $\mathrm{Si}$ surface on the crystal lattice of the particle. Ramakrishna and Friesner have calculated the effects of lattice contraction on the electronic structure of $\mathrm{H}$-terminated $\mathrm{Si}$ particles as a function of size, and they find the effect to be large, especially in the case of the (indirect) $X-\Delta$ tran- 
sition. We have grown six films of various sized particles in the size range $120-45 \AA$ and have measured the $x$-ray powder diffraction (XPD) features as a function of average particle size. XPD linewidths were consistent with estimated particle size distributions as measured by transmission electron microscopy (TEM). XPD peak positions fitted to the bulk Si diamond lattice constant with an accuracy of $\approx 0.2 \%$. Thus, hydrogen does appear to be a good surface terminator, and lattice contraction effects are not important. The XPD signal was too weak to measure the lattice constants of the smallest particles discussed here $(25 \AA)$. However, TEM electron diffraction measurements on these particles indicate that they are also diamond lattice, although the experimental uncertainty of this measurement is about 1\%. XPD measurements on all size ranges reveal no evidence for the presence of amorphous material.

For the spatially resolved EELS measurements, particles were collected onto a holey carbon grid with 7 SCCM $5 \% \mathrm{Si}_{2} \mathrm{H}_{6} / \mathrm{He}$ flow rates for a period of $10 \mathrm{~s}$ under conditions meant to optimize a broad size distribution. Since the measurements are done on single particles, a broad size distribution actually makes the experiment easier. The grid was removed from the vacuum chamber and placed in a glass vacuum flask. The flask was immediately evacuated, back filled with $\mathrm{Ar}$, and stored until loading in the UHV EELS apparatus. The total exposure of the grid to air amounted to less than $1 \mathrm{~min}$.

The spatially resolved EELS spectra were recorded on a VG Microscopes HB501 scanning transmission electron microscope (STEM) fitted with a Wien filter electron spectrometer [11]. The instrument was operated at 120 $\mathrm{kV}$ using a beam current of $0.5-1.0 \mathrm{nA}$ and a probe diameter of about $4 \AA$. The spectra were obtained with an energy resolution of $0.35 \mathrm{eV}$ and, in some cases, sharpened by deconvolution to $0.25 \mathrm{eV}$ [12]. Single particles were visually inspected to determine their diameter, then Si $2 p \rightarrow C B$ (conduction band) spectra were acquired for a probe position as close to the central axis of the particle as possible. All particles probed were approximately spherical in morphology.

Figure 1 shows representative spectra from several particles. Intensities have been normalized to enhance shape comparisons. We have modeled the bulk $s$ - $d$-projected final density of states (DOS) using three major components: parabolic band edges beginning at $\Delta_{1}$ and $L_{1}$, and back-to-back saddle point singularities to approximate the rather flat band at $L_{3}$. We further multiply these contributions by a factor which forces each DOS contribution to zero in a smooth manner as band maxima or dipole-forbidden positions in the $\mathrm{Si}$ Brillouin zone are approached. The DOS is therefore a sum of terms similar to [9]

$$
D(E)=\frac{m^{*}}{\pi^{2} \hbar^{2}}\left(E-E_{\mathrm{th}}\right)^{1 / 2} \theta\left(E-E_{\mathrm{th}}\right) F,
$$

where $m^{*}$ is the effective mass, $E_{\mathrm{th}}$ is the threshold ener912

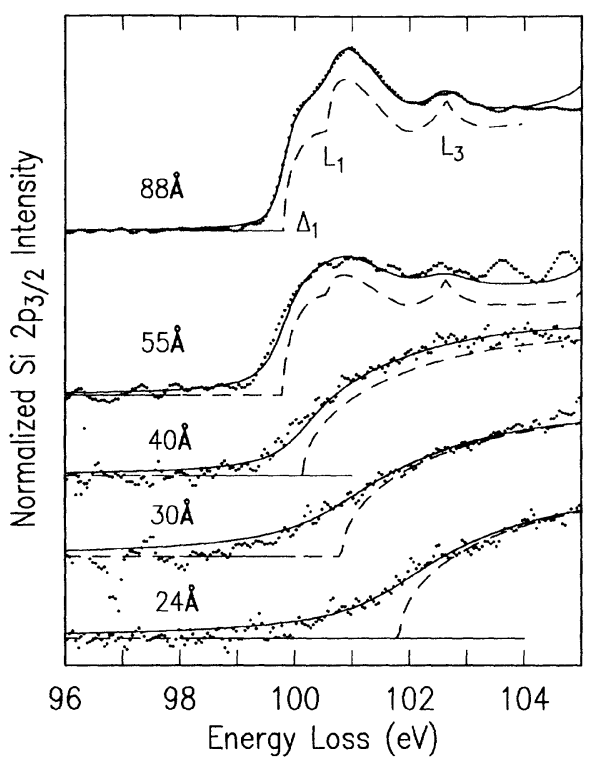

FIG. 1. Normalized EELS spectra as a function of particle diameter. The spectra have been modeled by sets of parabolic band edges corresponding to final states at $\Delta_{1}, L_{1}$, and $L_{3}$. Below $50 \AA$, the edge shape changes to a single parabolic component and shifts upwards in energy. As particle size decreases, damping by conduction electron collisions with the particle surface becomes visible.

gy, and $\theta(E)$ is the step function. $F$ is given by

$$
F=\left|\frac{E_{\max }-E}{E_{\max }-E_{\mathrm{th}}}\right|^{a} \theta\left(E_{\max }-E\right) .
$$

$E_{\max }$ is the maximum extent of $\mathcal{D}(E)$, and $\alpha$ is 1 for a dipole-allowed termination and 2 for a dipole-forbidden termination. Both $\Delta_{1}$ and $L_{1}$ contributions have two components, since they may terminate at dipole-allowed $(W)$ or dipole-forbidden $\left(\Gamma_{2^{\prime}}\right.$ for $L_{1}$ and $\Gamma_{1,5}$ for $\left.\Delta_{1}\right)$ positions, depending on crystal direction. Thus, $\Delta_{1}$ is characterized mainly by a parabolic edge, which terminates at a band maximum at $W . L_{1}$, on the other hand, resembles a peak because it mainly terminates at the dipole-forbidden $\Gamma_{2^{\prime}}$ position at fairly low energy. The relatively flat band at $L_{3}$ may be treated like two back-to-back saddle points forming a peak that terminates largely at the symmetryforbidden $\Gamma_{1,5}$ position which lies below $L_{3}$,

$$
\mathcal{D}(E)=\frac{m^{*}}{\pi^{2} \hbar^{2}}\left[1-\left|\left(E-E_{\mathrm{sp}}\right)\right|^{1 / 2}\right] F,
$$

where $E_{\mathrm{sp}}$ is the saddle point energy. $F$ is defined as

$$
F=\left(1-\left|\frac{E-E_{\mathrm{sp}}}{E_{\mathrm{sp}}-E_{\min }}\right|\right) \theta\left(E-E_{\mathrm{min}}\right),
$$

where $E_{\min }$ is the energy of the $\Gamma_{1,5}$ position. The modeled DOS is then a sum of terms like these for $\Delta_{1}, L_{1}$, and $L_{3}$. Finally, this DOS is inserted into the inelastic scattering theory to include effects due to core excitonic distortion, lifetime broadening, and instrumental energy 
resolution [13].

We find that the results for particles of diameter greater than $50 \AA$ are indistinguishable from those obtained in thin $\mathrm{Si}$ foils. These results are characterized by unshifted positions of $\Delta_{1}, L_{1}$, and $L_{3}$, plus a gradual damping of each contribution caused by the proximity of the Si surfaces. Thus, the bulk damping factor $\hbar \gamma=0.1$ $\mathrm{eV}$ for thick silicon, but increases to about $0.5 \mathrm{eV}$ for a 55 $\AA$ particle. This behavior closely resembles that found in the past for damping of bulk plasmons in small aluminum spheres [14].

Below $50 \AA$ the edge shape changes abruptly to a single parabolic band having no obvious upper limit. The damping continues to increase, becoming as much as 2 $\mathrm{eV}$ in the smallest particles, and the lower onset of the edge shifts dramatically to higher energy. In the absence of identifiable changes in the lattice constant of the $\mathrm{Si}$, we must deduce that the shift is caused by conduction band changes rather than by a shift in the $2 p$ core level. We plot the measured positions of the band onset as a function of particle diameter in Fig. 2. This behavior is in contrast to recent soft $\mathrm{x}$-ray absorption results for porous $\mathrm{Si}$ where an edge shift was observed without a gross change in its overall shape [15].

In an effective mass treatment for the motion of a conduction electron in the crystal, the electron wave function may be represented as a product of crystal Bloch waves with an envelope function, $\psi(r)$. This function is then a solution of an effective mass Schrödinger equation [16]

$$
-\frac{\hbar^{2}}{2 m^{*}} \nabla^{2} \psi+V \psi=E \psi
$$

where, in this case, $V$ is constant (taken to be zero), $m^{*}$ is the electron effective mass, and $E$ is the kinetic energy of the conduction electron characterized by the envelope

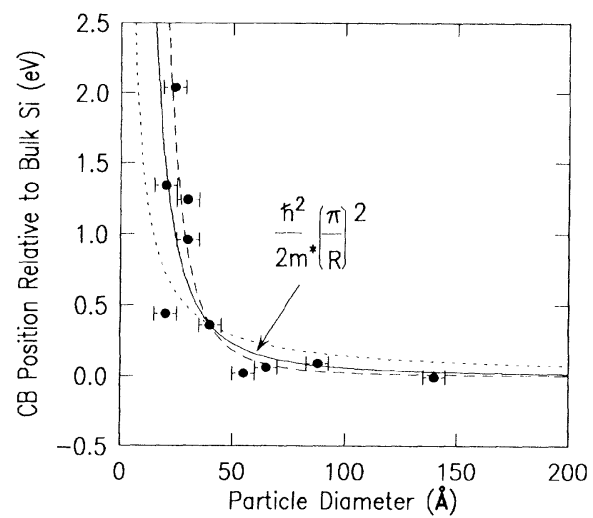

FIG. 2. Plot of the energy shift of the DOS edge onset as a function of particle diameter. The solid line shows the expected behavior for an effective-mass-like quantization of electrons in the particle. $1 / R$ (dotted line) and $1 / R^{3}$ (dashed line) behaviors are shown for comparison, normalized at $40 \AA$. We can rule out $1 / R$ behavior, but not $1 / R^{3}$. function, $\psi$. Within a bounded sphere, the solutions of this equation take the form of spherical Bessel functions, $J_{l}\left(k_{l}^{j} r / R\right)$ where $R$ is the radius of the sphere and $k_{l}^{j}$ is the $j$ th zero of the Bessel function $J_{l}(z)$. The kinetic energy of the electron described in this way is given by the standard form

$$
E(k)=\frac{\hbar^{2}}{2 m^{*}} k^{2}
$$

with the values of $k$ quantized by the boundary conditions requiring that $\psi(R)=0$. Then $k=k_{l}^{j} / R$. We plot the prediction of Eq. (6) in Fig. 2 using the lowest available mode $\left(k_{0}^{1}=\pi\right)$ and an average effective mass of $0.26 \mathrm{~m}$ for silicon [17]. For comparison we also plot predictions proportional to $R^{-1}$ and $R^{-3}$ normalized at $40 \AA$. We find that the best agreement is with the $1 / R^{2}$ prediction, although we cannot rule out the $1 / R^{3}$ behavior. In addition, the magnitude of the energy shift is given nicely by the simple expression of Eq. (6).

Figure 3 is a plot of the scattering cross section relative to bulk silicon as a function of particle size. This has been integrated over a $2 \mathrm{eV}$ wide window beginning at the edge onset. As found in the earlier work on thin films, the cross section is independent of thickness (diameter) down to about $50 \AA$, in spite of the decrease in visibility of the fine structure due to damping. When the single parabolic edge shape develops below $50 \AA$, the cross section also begins to increase. Initially, this can be explained by a relaxation of the normally $p$-like symmetry at the Brillouin zone center-allowing the $s$ - and $d$ projected effective mass DOS to increase without limit towards higher energies. But below $40 \AA$ the cross section continues to increase, even though the shape of the edge does not undergo further changes. This suggests that larger regions of phase space in the Brillouin zone be-

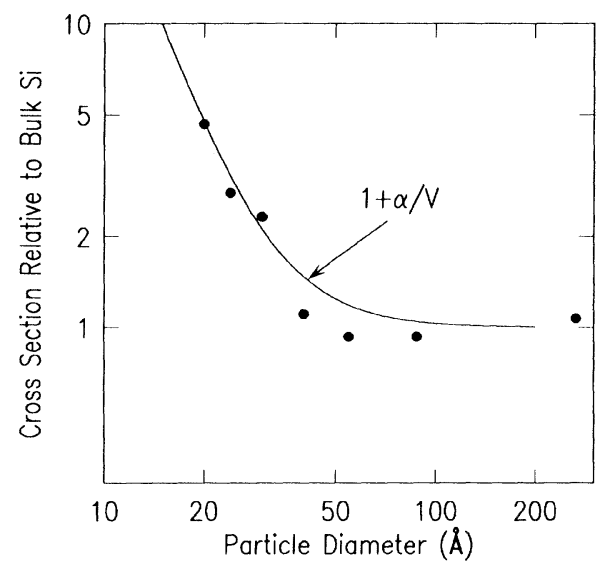

FIG. 3. The partial cross section enhancement relative to bulk Si. The solid line provides an inverse volume behavior for comparison. This behavior possibly signals a relaxation of the dipole selection rules, allowing a direct transition from the Si $2 p$ core level to the Brillouin zone center. 
come dipole accessible due to quantization as the particles become smaller. Most of the bulk silicon conduction bands have $p$-like symmetry, so that they do not normally contribute in the bulk scattering experiment using the $2 p$ core level as an initial state. Therefore, any relaxation of the dipole selection rules would almost certainly result in a large enhancement of the scattering cross section. For comparison, we have plotted an inverse volume dependence in the figure, but note that at present we have no detailed understanding of the shape of the enhancement.

In summary, we have produced $\mathrm{H}$-terminated silicon clusters in the 20-500 $\AA$ range, and have obtained conduction band information from individual particles as a function of their diameter. We find strong changes in the edge position, shape, and intensity below $50 \AA$ diameter, and believe that these signal size quantization of the conduction electrons. A simple effective mass theory using the average bulk value of the mass for silicon adequately explains the shift of the edge position, although we cannot rule out absolutely a shift that might be an inverse function of the particle volume.

[1] H. Takagi, H. Ogawa, Y. Yamazaki, A. Ishizaki, and T. Nakagiri, Appl. Phys. Lett. 56, 2379 (1990).
[2] K. A. Littau, P. J. Szajowski, A. J. Muller, A. R. Kortan, and L. E. Brus, J. Phys. Chem. 97, 1224 (1993).

[3] J. R. Heath and J. M. Jasinski, Mater. Res. Soc. Symp. Proc. 256, 117 (1992).

[4] L. T. Canham, Appl. Phys. Lett. 57, 1046 (1990).

[5] M. S. Hybertson, Mater. Res. Soc. Symp. Proc. 256, 179 (1992).

[6] M. V. Ramakrishna and R. A. Friesner, J. Chem. Phys. 96, 873 (1992).

[7] S. Glutsch and F. Bechstedt, Phys. Rev. B 47, 4315 (1993).

[8] G. D. Sanders and Y-C. Chang, Phys. Rev. B 45, 9202 (1992).

[9] P. E. Batson, Ultramicroscopy 50, 1 (1993).

[10] J. M. Jasinski and F. K. LeGoues, Chem. Mater. 3, 989 (1991).

[11] P. E. Batson, Rev. Sci. Instrum. 57, 43 (1986); 59, 1132 (1988).

[12] P. E. Batson, D. W. Johnson, and J. C. H. Spence, UItramicroscopy 41, 137 (1992).

[13] P. E. Batson and J. Bruley, Phys. Rev. Lett. 67, 350 (1991); P. E. Batson, Phys. Rev. B 47, 6898 (1993).

[14] P. E. Batson, Solid State Commun. 37, 477 (1980).

[15] T. van Buuren, Y. Gao, T. Tiedje, J. R. Dahn, and B. M. Way, Appl. Phys. Lett. 60, 3013 (1992).

[16] J. M. Luttinger and W. Kohn, Phys. Rev. 97, 869 (1955).

[17] T. Takagahara and K. Takeda, Phys. Rev. B 46, 15578 (1992). 